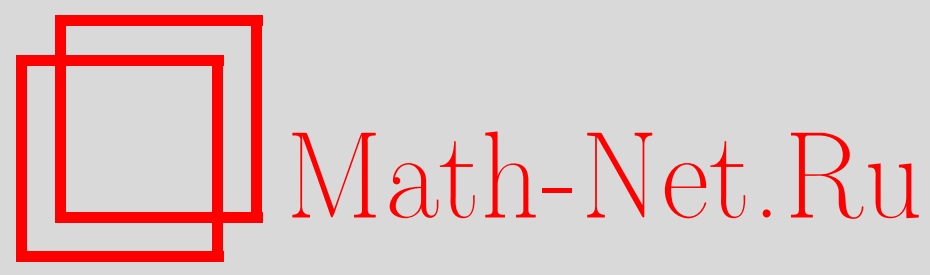

В. Е. Тараканов, Оценки числа независимости гиперграфа и гипотеза Райзера, Матем. заметки, 1997, том 61, выпуск $6,873-883$

DOI: https://doi.org/10.4213/mzm1571

Использование Общероссийского математического портала Math-Net.Ru подразумевает, что вы прочитали и согласны с пользовательским соглашением http://www.mathnet.ru/rus/agreement

Параметры загрузки:

IP : 3.85 .5 .30

26 апреля 2023 г., 14:03:26

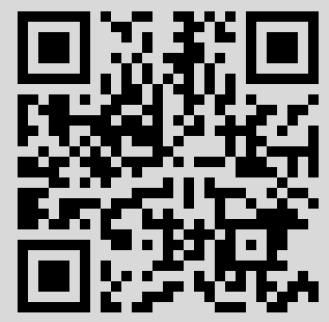




\section{ОЦЕНКИ ЧИСЛА НЕЗАВИСИМОСТИ ГИПЕРГРАФА И ГИПОТЕЗА РАЙЗЕРА}

\section{В. Е. Тараканов}

Пусть $H$ - гиперграф с $n$ вершинами, $m$ ребрами, числом вершин в ребре не больше $r \geqslant 2$ и степенью вершины $\delta \geqslant 2 ; \tau(H)$ - его трансверсальное число, $\mu(H)$ - число независимости (максимальное число попарно не пересекающихся peбер с $r$ вершинами в ребре). Известная оценка $\mu \geqslant(\delta n-(r-1) m) /(\delta r-r+1)$ усиливается в случае, когда $H$ - псевдограф̆ с максимальной степенью вершины $\Delta$ при $2 \delta-2 \geqslant \Delta$ (аналог соответствующего результата для графов), и применяется для доказательства известной гипотезы Райзера для $r$-дольных $r$-равномерных гиперграфов $H: \tau(H) \leqslant(r-1) \mu(H)$, а именно, устанавливается справедливость этого неравенства в случае, когда $H \quad \delta$-регулярен, $2 \leqslant \delta \leqslant r-1$.

Библиограффия: 6 названий.

1. Введение. Пусть $V$ - конечное множество с $n$ элементами и $E$ - система из $m$ его подмножеств. В этом случае говорим о гиперграфе $H=(V, E)$ с $n$ вершинами (элементами из $V$ ) и $m$ ребрами (подмножествами из $E$ ). Обозначим через $r=r(H)$ максимальное число вершин в ребре гиперграфа $H$. Через $\delta=\delta(H)$ обозначим число ребер, которым может принадлежать вершина гиперграфа $H$ (т.е. минимальную степень вершины в $H)$. Числом независимости гиперграфа $H$ называется наибольшеевозможное число $\mu(H)$ попарно не пересекающихся ребер с $r$ вершинами. (Совокупность попарно не пересекающихся ребер мощности $r$ назьвается паросочетанием (matching) в $H$, а $\mu(H)$ иногда назьвают числом паросочетания.) Это число является существенной характеристикой гиперграфа и играет значительную роль в экстремальной теории гиперграфов. Поэтому представляют интерес нижние границы этого числа для классов гиперграфов, выделяемых тем или иным условием относительно его параметров. Другие структурные константы, интенсивно исследуемые в экстремальной теории гиперграфов, - это

1) реберное число покрытия $\varepsilon(H)$ - минимальное число ребер, объединение которых совпадает с $V$, и

2) трансверсальное число $\tau(H)$ - минимальное число вершин в таком подмножестве $T \subseteq V$, что любое число ребер содержит вершину из $T$ (такое множество $T$ называют трансверсалью).

Работа выполнена при финансовой поддержке Российского фонда фундаментальных исследований, грант № 96-01-00531. 
С гиперграфом $H=(V, E)$ связьвается $(0,1)$-матрица $A(H)$ - его матрища инцидентности. Пусть элементы множества $V$ и подмножества системы $E$ как-либо перенумерованы:

$$
V=\left\{v_{1}, v_{2}, \ldots, v_{n}\right\}, \quad E=\left\{e_{1}, e_{2}, \ldots, e_{m}\right\} .
$$

Тогда $A(H)=\left(a_{i j}\right)$ есть матрица размера $m \times n$, т.е. с $m$ строками и $n$ столбцами, такая, что

$$
a_{i j}= \begin{cases}1, & \text { если } v_{j} \in E_{i}, \\ 0 & \text { в противном случае; }\end{cases}
$$

имеем также $\sum_{j=1}^{n} a_{i j} \leqslant r, i=1,2, \ldots, m$. Матрица инцидентности определяется гиперграфом с точностью до перестановочной эквивалентности, т.е. каждому гиперграфу $H$ соответствует класс $(0,1)$-матриц, каждая из которых получается из другой перестановками строк и столбцов; любую из этих матриц можно рассматривать как матрицу инцидентности $A(H)$ гиперграфа $H$. Структурньг константам гиперграфа $H$ соответствуют инварианты матрицы $A(H)$ относительно перестановочной эквивалентности. Так $\varepsilon(H)$ есть не что иное, как глубина $\varepsilon_{A}$ матрицы $A=A(H)$, т.е. наименьшее возможное число строк в $A(H)$ таких, что сумма элементов в каждом столбце образованной ими подматрицы положительна. Число $\tau(H)$ есть ширина $A$, т.е. наименьшее число столбцов в $A$ таких, что сумма элементов в каждой строке образованной ими подматрицы положительна.

Определим строчны й $i$-ранг, или просто $i$-ранг, $(0,1)$-матрицы $A$ с максимальньм числом $r$ единиц в строке как наибольшее возможное число попарно ортогональных строк с $r$ единицами (строки при этом понимаются как $n$-мерные действительные векторы, $n$ - число столбцов в $A$ ). Число независимости $\mu(H)$ гиперграфа $H$ равно величине строчного $i$-ранга его матрицы инцидентности $A(H)$, и всякая оценка снизу $i$-ранга $A(H)$ есть оценка для $\mu(H)$.

В работах [1], [2] нами фактически была получена следующая оценка снизу числа независимости $\mu(H)$ гиперграфа $H$.

Tеорема А. Пусть $H=(V, E)$ - гиперграф на множестве $V$ из $h$ әлементов, $m$ - число его ребер, $r$ - наибольшее число вериин в ребре. Тогда

$$
\mu(H) \geqslant \frac{\delta n-(r-1) m}{\delta r-r+1},
$$

где $\delta \geqslant 2$ - наименьшая степень вериинь в $H$.

Ясно, что оценка (1) справедлива для $r$-равномерны $x$ гиперграфов, т.е. таких, у которых все ребра состоят из одинакового числа $r$ вершин (их называют также $r$-графами). Оценка (1) была получена в матричных терминах. Она позволила развить метод изучения глубины $(0,1)$-матрищ (т.е. реберного числа покрытия гиперграфа) и дать для нее точные и асимптотические оценки [1], [2].

Различные применения в экстремальной теории гиперграфов делают актуальной задачу уточнения оценки (1) в тех или иных частных случаях. В п. 2 мы получим такую улучшенную оценку для случая, когда $H$ - псевдограф при заданной наименьшей $\delta$ и наибольшей $\Delta$ степени его вершины. Такого рода оценки числа независимости изучались для графов (см. [3, гл. 2]). Полученные нами границы - это обобщение некоторых из этих гранищ. 
Гиперграф $H$ назьвается $\delta$-регулярным, если всякая его вершина принадлежит в точности $\delta$ ребрам. Пусть $|X|$ обозначает число элементов в множестве $X$. Если в $r$-графе $H=(V, E)$ (т.е. в $r$-равномерном гиперграфе) множество вершин может быть разбито на $r$ равномощных подмножеств $V_{1}, V_{2}, \ldots, V_{r}$ таким образом, что $\left|e \cap V_{i}\right|=1$ для любого ребра $e \in E, i=1, \ldots, r$, то $H$ называется $r$-дольным $r$-әрафом. Гипотеза Райзера для $r$-дольных $r$-графов заключается в предположении, что для таких графов справедливо следующее неравенство:

$$
\tau(H) \leqslant(r-1) \mu(H) .
$$

Этой гипотезе посвящен ряд работ (см., например, [4], [5]). Она доказана лишь для небольших значений $\mu(H)$; известны [5] также некоторые оценки вида $\tau(H) \leqslant \alpha \mu(H)$, $2<\alpha<3$, при $r=3$. Мы используем для рассмотрения гипотезы Райзера оценку (1) и в п. 3 с ее помощью покажем справедливость этой гипотезы в классе $\delta$-регулярных $r$-графов при $2 \leqslant \delta \leqslant r-1$. Мы получим также оценку

$$
\tau(H) \leqslant\left(r-1+\frac{1}{r}\right) \mu(H)
$$

( $r$ произвольно) для $r$-дольных $r$-графов с $\Delta \leqslant r$ и числом ребер, равньм числу вершин.

\section{2. $i$-каноническая форма матрицы инцидентности псевдографа и оценки} его числа независимости. Псевдограф определяется аналогично графу, с той разнищей, что в нем допускаются петли и кратные ребра. Мы получим в этом пункте оценки снизу числа независимости для псевдографа $H \mathrm{c} \delta \geqslant 2$. Эти оценки, в отличие от общей оценки (1) для гиперграфов, учитьвают не только минимальную величину $\delta$, но также максимальную величину $\Delta$ степени вершины. При $\Delta=\delta$ они улучшают (1). При выводе оценок мы используем матрицу инцидентности псевдографа, оценивая фактически ее строчный $i$-ранг. Если $H$ - псевдограф с $n$ вершинами, $m$ ребрами, то его матрища инцидентности $A(H)$ - это $(0,1)$-матрица размера $m \times n$, все ее строчные суммы не превосходят 2 . Пусть $r, \delta$ - натуральные числа. Класс всех $(0,1)$-матриц размера $m \times n$ со строчными суммами, не превосходящими $r$, и столбцовьми суммами, не меньшими $\delta$, обозначим $\mathfrak{A}^{m, n}(\leqslant r, \geqslant \delta)$.

Введем еще несколько определений. Пусть $A$ - действительная неотрицательная матрица размера $m \times n$ и $r$ - максимальное число положительных элементов в ее строке, $0 \leqslant r \leqslant n$. Вектор-строки $l_{1}, l_{2}, \ldots, l_{k}$ матрищы $A$ называются независимыми, если скалярные произведения $\left(l_{i}, l_{j}\right)=0, i \neq j, i, j=1,2, \ldots, k$. Если $A$ - матрица инцидентности гиперграфа $H$, то совокупности ее независимых строк отвечает совокупность независимых ребер в $H$. Cтрочным $i$-базисом ненулевой неотрицательной матрицы $A$ назовем всякое максимальное по мошности множество ее независимых строк, каждая из которых имеет наибольшее возможное число положительных элементов. Строчный $i$-ранг $\mu(A)$ матрицы $A$ определяется как число строк в ее строчном $i$-базисе (см. п. 1$)$. Пусть $\mu(A)=0$ для нулевой матрищы $A$. Очевидно, что $\mu(A)$ не превосходит граничного ранга $\rho(A)$ неотрищательной матрищы $A$ (определяется аналогичнограничному рангу $(0,1)$-матрицы, см. [6]), а также ее ранга как матрицы над полем. 
Рассмотрим теперь некоторое стандартное представление матрицы инцидентности $A(H)$ псевдографа $H$ с $n$ вершинами и $m$ ребрами (см. [2]), которое назовем ее $i$-канонической формой. Матрица $A(H)$ принадлежит классу $\mathfrak{A}^{m, n}(\leqslant 2, \geqslant \delta)$, если $\delta$ - минимальная степень вершины в псевдографе. Представление, о котором идет речь, достигается перестановками строк и столбцов, приводящими к разбиению $A(H)=A$ на три горизонтальные полосы $(A)_{1},(A)_{2},(A)_{3}$. Пусть в псевдографе $H$ имеется ребро, не являющееся петлей. Возьмем в $A$ какой-либо $i$-базис $\mathscr{L}$, состоящий из $\mu$ строк с суммой 2 , где $\mu$ - (строчный) $i$-ранг $A$. В $(0,1)$-матрице $A=\left(a_{i j}\right)$ считаем, что строка $i$ покрывает столбец $j$, если $a_{i j}=1$. Переместим строки из $\mathscr{L}$ и покрытые ими столбцы на первые места. Образованную этими строками подматрицу размера $\mu \times n$ обозначим через $(A)_{1}$. Так как $\mathscr{L}-i$-базис, то подматрица, образованная теперь последними $n-\mu$ строками и последними $n-2 \mu$ столбцами, имеет в каждой строке не более одной единицы. Рассмотрим далее все строки матрищы, покрывающие столбцы с номерами $n-2 \mu+1, n-2 \mu+2, \ldots, n$. Для каждого из этих столбцов берем по порядку все покрывающие его строки и располагаем их подряд. Образованную таким способом подматрицу обозначим через $(A)_{2}$. Пусть $m_{2}$ - число строк в $(A)_{2}$. Если степень вершины в $H$ есть число из отрезка $[\delta, \Delta]$, то, очевидно, $\delta(n-2 \mu) \leqslant m_{2} \leqslant \Delta(n-2 \mu)$. Пусть последние $m_{3}=m-\left(\mu+m_{2}\right)$ строки образуют подматрицу $(A)_{3}$. Из построения ясно, что $(A)_{1}$ соответствуют ребра некоторого максимального паросочетания $\mathscr{L}$ псевдографа, $(A)_{2}$ - все ребра, проходящие через вершины, не принадлежащие ребрам из $\mathscr{L}$, и $(A)_{3}$ - ребра, не входящие в $\mathscr{L}$, но соединяющие вершины, принадлежащие ребрам из $\mathscr{L}$. Подматрицу $(A)_{2}$ мы далее подразделяем на горизонтальные отсеки $(A)_{2}^{j}$, каждьй из которых состоит из строк, покрьвающих один и тот же из последних $n-2 \mu$ столбцов, $j=n-2 \mu+1, \ldots, n$. Подматрица $(A)_{2}$ пуста, если $\mu=n / 2$. Описанное представление матрицы инцидентности $A(H)$ псевдографа $H$ назьвается ее (строчной) i-канонической формой.

В этом пункте мы рассматриваем псевдографы $H=(V, E),|V|=n,|E|=m$, с фиксированньми минимальными $\delta$ и максимальными $\Delta$ степенями вершин. Соответствующий подкласс $(0,1)$-матриц, которому принадлежат матрицы инцидентности таких псевдографов, состоит из матриц размера $m \times n$, у которых строчные суммы не превосходят 2 , а все столбцовые суммы принадлежат фиксированному отрезку $[\delta, \Delta]$. Такой подкласс обозначим через $\mathfrak{A}^{m, n}(\leqslant 2,[\delta, \Delta])$. Мы фактически оценим снизу строчньй $i$-ранг матрицы из $\mathfrak{A}^{m, n}(\leqslant 2,[\delta, \Delta])$. Будем рассматривать матрицы из этого класса в $i$-канонической форме. Найдем сначала некоторые свойства таких матриц.

Предполагаем в дальнейшем, что $A$ - матрища из $\mathfrak{A}^{m, n}(\leqslant 2,[\delta, \Delta])$ в $i$-канонической форме при заданном $i$-базисе $\mathscr{L}$. Если $l$-строка в $A$ с двумя единицами, то покрытые ею столбцы обозначаем $c^{1}(l), c^{2}(l)$; строку $l$ из $\mathscr{L}$ назовем строкой типа $T_{\gamma}$, если в $(A)_{3}$ имеется в точности $\gamma$ единиц, принадлежащих столбцам $c^{1}(l), c^{2}(l)$. Существуют две возможности:

1) оба столбца имеют единицы в $(A)_{2}$;

2) по крайней мере, один из них не имеет единиц в $(A)_{2}$.

В соответствии с этим строку $l$ из $\mathscr{L}$ типа $T_{\gamma}$ будем называть строкой тuпа $T_{\gamma}^{(1)}$ и $T_{\gamma}^{(2)}$ (или просто тuпа $T^{(1)}$ и тuna $T^{(2)}$, если значение $\gamma$ для нас несущественно). В этом же смысле будем говорить о $T_{\gamma}$-строке, $T_{\gamma}^{(1)}$ - или $T_{\gamma}^{(2)}$-строке, $T^{(1)}$ - или $T^{(2)}$-строке. Столбец, покрытьй строкой $l \in(A)_{1}$, назовем длинным, если он не имеет единищ в $(A)_{2}$, 
и коротким - в противном случае. Ясно, что у $T^{(1)}$-строки оба столбца короткие, а у $T^{(2)}$-строки хотя бы один столбец длинный.

Лемма 1. Пусть $l \in \mathscr{L}-T^{(1)}$-строка. Тогда все единиць столбцов $c^{1}(l) u$ $c^{2}(l)$, располохсенные в $(A)_{2}$, сосредоточены в каком-либо одном отсеке этой подматрииы.

ДокАЗАТЕльство. Пусть это не так. Тогда по определению $T^{(1)}$-строки в $(A)_{2}$ найдутся две строки $t_{1}$ и $t_{2}$ такие, что $t_{1}$ покрьвает $c^{1}(l), t_{2}$ покрывает $c^{2}(l)$ и $\left(t_{1}, t_{2}\right)=0$. Таким образом, строки в множестве $(\mathscr{L}-\{l\}) \cup\left\{t_{1}, t_{2}\right\}$ независимы. Это противоречит тому, что $\mathscr{L}-i$-базис.

Лемма 2. Каждая строка $l$ i-базиса $\mathscr{L}$ имеет mun $T_{\gamma}$, əде $2 \Delta-2 \geqslant \gamma \geqslant \max \{0$, $2 \delta-\Delta-2\}$. Если $\gamma=2 \delta-\Delta-2>0 u l-T_{\gamma}$-строка, то она $T^{(1)}$-строка $u$ столбиь $c^{1}(l), c^{2}(l)$ имеют в $(A)_{2}$ в общей сложности $\Delta$ единии, которые все сосредоточены в одном отсеке.

ДоказАтельство. Так как $A \in \mathfrak{A}(\leqslant 2,[\delta, \Delta])$, имеем $\gamma+2 \leqslant 2 \Delta$ и $\gamma \leqslant 2 \Delta-2 . \mathrm{C}$ другой стороны, $c^{1}(l)$ и $c^{2}(l)$ вместе имеют не менее $2 \delta$ единиц, из которых в $(A)_{2}$ расположены $2 \delta-2-\gamma$ единиц. Наибольшее число единиц этих столбцов, которые могут "уместиться" в одном отсеке, равно $\Delta$. Если бы вьполнялось $2 \delta-2-\gamma>\Delta$, то какая-либо пара единиц, одна из которых принадлежит $c^{1}(l)$, а другая $-c^{2}(l)$, находились бы в разньх отсеках подматрицы $(A)_{2}$. Тогда, рассуждая так же, как при доказательстве леммы 1 , мы пришли бы к противоречию с тем, что $\mathscr{L}-i$-базис. Итак, $2 \delta-\gamma-2 \leqslant \Delta$ и $\gamma \geqslant 2 \delta-\Delta-2$. Пусть теперь $2 \delta-\Delta-2>0$ и $\gamma=2 \delta-\Delta-2$. Если бы один из покрытых строкой $l$ типа $T_{\gamma}$ столбцов был длинным, т.е. все его единицы принадлежали бы $(A)_{3}$, то мы имели бы $\delta-1 \leqslant \gamma=2 \delta-\Delta-2$, т.е. $\delta \geqslant \Delta+1$, что невозможно. Таким образом, $l-T^{(1)}$-строка, и по лемме 1 все $2 \delta-2-\gamma=\Delta$ единиц столбцов $c^{1}(l), c^{2}(l)$, находящихся в $(A)_{2}$, сосредоточены в одном отсеке.

Для натурального числа $v$ назовем строки $l$ и $l^{\prime} i$-базиса $\mathscr{L} v$-связанными, если в $(A)_{3}$ сушествует в точности $v$ строк $t$ со свойством $(l, t)=\left(l^{\prime}, t\right)=1$.

ЛЕмма 3. Пусть $l, l_{1}-$ две строки $i$-базиса $\mathscr{L}$. Положим $\gamma=2 \delta-\Delta-2 u$ предположим, что $\gamma>0$ u $l-T_{\gamma}$-строка, $l_{1}-T_{\gamma_{1}}$ сттрока, где $\gamma_{1} \leqslant 2 \delta-3$. Если строки $l$ и $l_{1} v$-связаны, то $l_{1}-T^{(2)}$-строка и все $v$ строк, связываюших $l u l_{1}$, покрывают короткий столбеч, строки $l_{1}$.

ДокАЗАТЕльство. Так как $\gamma_{1}<2 \delta-2$, то хотя бы один из столбцов $c^{1}\left(l_{1}\right), c^{2}\left(l_{1}\right)$ короткий, скажем, столбец $c^{1}\left(l_{1}\right)$. Допустим, что существует связующая строка $t$, которая покрывает $c^{2}(l)$ и $c^{2}\left(l_{1}\right)$. По лемме $2 l-T^{(1)}$-строка, и все $\Delta$ принадлежащих $(A)_{2}$ единиц обоих столбцов, покрытых строкой $l$, лежат в одном отсеке $(A)_{2}^{j}$ подматрицы $(A)_{2}$. Пусть единица короткого столбца $c^{1}\left(l_{1}\right)$ лежит в отсеке $(A)_{2}^{j}$. Имеем $j_{1} \neq j$. Тогда, очевидно, в $(A)_{2}$ найдутся такие две ортогональные строки $u, u_{1}$, что $u$ принадлежит $(A)_{2}^{j}$ и покрьвает $c^{1}(l), u_{1}$ лежит в $(A)_{2}^{j_{1}}$ и покрывает $c^{1}\left(l_{1}\right)$. Следовательно, множество $\left(\mathscr{L}-\left\{l, l_{1}\right\}\right) \cup\left\{t, u, u_{1}\right\}$ состоит из попарно ортогональных строк. Но $\mathscr{L}-$ это $i$-базис, и мы приходим к противоречию.

ЛЕмма 4. Пусть $\gamma=2 \delta-\Delta-2>0 u l-T_{\gamma}$-строка, $v$-связанная $c T_{\gamma_{1}}$-строкой $l_{1}$. Tогда $\gamma_{1} \geqslant v+\delta-1$. 
ДокАЗАТЕЛЬство. Пусть $\gamma_{1} \leqslant 2 \delta-3$. Тогда по лемме $3 l_{1}-T^{(2)}$-строка, и ее короткий столбец содержит не менее $v$ единищ в $(A)_{3}$. Поэтому $\gamma \geqslant v+\delta-1$.

Пусть $\gamma_{1} \geqslant 2 \delta-2$. Тогда в $(A)_{3}$ находится, помимо $v$, еще не меньше $2 \delta-2-v$ единиц столбцов, покрьваемых $l_{1}$. Но $v \leqslant \gamma \leqslant 2 \delta-\Delta-2$ и $2 \delta-2-v \geqslant \Delta>\delta-1$, т.е. $\gamma_{1} \geqslant 2 \delta-2>v+\delta-1$.

Полученные результаты дают возможность оценить снизу число единиц в подматрице $(A)_{3}$ матрицы $A \in \mathfrak{A}^{m, n}(\leqslant 2,[\delta, \Delta])$, заданной в $i$-канонической форме.

Теорема 1. Пусть $m, n, \delta, \Delta, 2 \leqslant \delta \leqslant \Delta,-$ натуральные числа, $H$ - псевдограф c $n$ вериинами, т ребрами, у которого степень $d(x)$ - число из отрезка $[\delta, \Delta]$ для всякой вершины $x$. Пусть, далее, $A(H)$ - матрица инцидентности $H, \mu-$ его число независимости. Тогда $A(H)$ әквивалентна матрице $A$ в $i$-канонической форме, у которой

1) при любом $\Delta$

$$
\sigma_{1}\left((A)_{3}\right) \geqslant \mu(2 \delta-\Delta-2),
$$

2) при нечетном $\Delta$

$$
\sigma_{1}\left((A)_{3}\right) \geqslant \mu(2 \delta-\Delta-1),
$$

где $\sigma_{1}(X)$ - число единии, в матрице $X$.

ДоКАЗАТЕЛЬСТВо. Ясно, что $A(H) \in \mathfrak{A}^{m, n}(\leqslant 2,[\delta, \Delta])$ и $\mu$ - строчньй $i$-ранг любой матрицы, эквивалентной $A(H)$. Если $2 \delta-\Delta-2 \leqslant 0$, то (3) и (4) выполняются тривиальньм образом. Поэтому далее предполагаем, что $2 \delta-\Delta-2>0$. Возьмем сначала произвольный строчньй $i$-базис $\mathscr{L}$ в $A(H)$ и указанным вьше способом перейдем от $A(H)$ к эквивалентной ей матрице $A$ в $i$-канонической форме относительно $\mathscr{L}$. Неравенство (3) следует непосредственно из леммы 2 . Предполагаем далее, что $\Delta$ нечетно. Обозначим $2 \delta-\Delta-2$ через $\gamma$. Если $\mathscr{L}$ не содержит $T_{\gamma}$-строки, то $(4)$ следует из леммы 2 . Поэтому предполагаем, что $\mathscr{L}$ содержит $T_{\gamma}$-строку $l$. По лемме 2 столбцы $c^{1}(l), c^{2}(l)$ имеют в $(A)_{2}$ в общей сложности $\Delta$ единищ, которые находятся в одном отсеке $\left(A_{2}\right)^{j}, n-2 \mu+1 \leqslant j \leqslant n$. Обозначим через $c$ столбец с номером $j$. Не уменьшая общности, можем предположить, что покрывающие $c$ строки $t_{1}, t_{2}, \ldots, t_{\Delta}$ расположены так, что $c^{1}(l)$ имеет единищы в строках $t_{1}, t_{2}, \ldots, t_{h}$, a $c^{2}(l)$ - в строках $t_{h+1}, \ldots, t_{\Delta}$ отсека $(A)_{2}^{j}$, при этом $h \geqslant(\Delta+1) / 2$.

Строки в $(A)_{3}$, покрьвающие $c^{2}(l)$, обозначим $t_{1}^{\prime}, t_{2}^{\prime}, \ldots, t_{h^{\prime}}^{\prime}$. Так как число единиц в столбце не меньше $\delta$, имеем $h^{\prime} \geqslant \delta-1-(\Delta-h)=h+\delta-\Delta-1$. Покажем теперь, что

$$
0<h^{\prime}<h
$$

Пусть от противного $h^{\prime} \leqslant 0$. Тогда имеем

$$
h \leqslant \Delta+1-\delta \Longrightarrow \frac{\Delta+1}{2} \leqslant \Delta+1-\delta \Longrightarrow 2 \delta-\Delta-1 \leqslant 0 .
$$

Но по предположению $0<2 \delta-\Delta-2$, т.е. $2 \delta-\Delta-1>1$. Мы приходим к противоречию. Итак, $0<h^{\prime} \leqslant \gamma=2 \delta-\Delta-2$. Далее, $h^{\prime}+1+\Delta-h \geqslant \delta$, следовательно, $h^{\prime} \leqslant h+\delta-\Delta-1<h$. Оба неравенства в (5) доказаны. 
Для иллюстрации наших обозначений и построений можно, например, рассмотреть следующий чертеж, относящийся к случаю $\delta=\Delta=5, \gamma=3, h=3, h^{\prime}=2$ :

$(A)_{2}^{j}\left\{\begin{array}{cccc}c^{1}(l) & c^{2}(l) & c \\ t_{1} & 1 & 1 & \\ t_{2} & 1 & & 1 \\ t_{3} & 1 & & 1 \\ t_{4} & & 1 & 1 \\ t_{5} & & 1 & 1 \\ t_{1}^{\prime} & 1 & 1 & \\ t_{2}^{\prime} & & 1 & \end{array}\right.$

Проведем следующие преобразования в $A$ :

1) поменяем местами столбцы $c$ и $c^{2}(l)$;

2) поменяем местами строки $l$ и $t_{h}$;

3 ) поменяем местами строки $t_{1}, t_{2}, \ldots, t_{h^{\prime}}$ и $t_{1}^{\prime}, t_{2}^{\prime}, \ldots, t_{h^{\prime}}^{\prime}$ (напомним, что $h^{\prime}<h$, cм. (5));

4) если $h^{\prime}+1<h$, то строки $t_{h^{\prime}+1}, t_{h^{\prime}+2}, \ldots, t_{h-1}$ переставим на места ниже строк, входящих в $(A)_{2}$.

В результате получим матрицу, у которой

a) на месте строки $l$ вновь будет строка с двумя единицами на тех же местах;

б) на месте столбца $c$ будет столбец с идущими подряд $\Delta-\left(h-h^{\prime}-1\right)$ единицами;

в) покрытые новой строкой $l$ столбцы имеют в $(A)_{3}$ не менее

$$
2 h^{\prime} \geqslant 2(h+\delta-\Delta-1) \geqslant 2 \frac{\Delta+1}{2}+2 \delta-2 \Delta-2=2 \delta-\Delta-1
$$

единиц, т.е. строка $l$ теперь имеет тип $T_{\gamma_{1}}$ с $\gamma_{1} \geqslant 2 \delta-\Delta-1$.

Покажем, что проведенные преобразования не могут привести к появлению новой $T_{\gamma}$-строки. Действительно, помимо $c^{1}(l), c^{2}(l)$ и $c$ преобразования затрагивают лишш столбцы, покрытые строками, связанньми со строкой $l$. Пусть $l^{\prime}$ была $v$-связана с $l$. Тогда по лемме $4 l^{\prime}$ есть $T_{\gamma^{\prime}}$-строка с $\gamma^{\prime} \geqslant v+\delta-1$. В результате преобразований из $(A)_{3}$ в $(A)_{2}$ могут переместиться не более $v$ единищ столбцов $c^{1}\left(l^{\prime}\right), c^{2}\left(l^{\prime}\right)$. Поэтому $l^{\prime}$ становится строкой типа $T_{\gamma_{1}^{\prime}}$ с $\gamma_{1}^{\prime} \geqslant \gamma-v \geqslant \delta-1 \geqslant 2 \delta-\Delta-1$. Таким образом, новой $T_{\gamma}$-строки не появляется, и мы пришли к новому $i$-базису, для которого число $T_{\gamma}$-строк на одну меньше, чем в прежнем. Поступая так же поочередно с каждой из оставшихся $T_{\gamma}$-строк, мы придем, наконец, к $i$-базису без $T_{\gamma}$-строк. Для этого $i$-базиса $\sigma_{1}\left((A)_{3}\right) \geqslant \mu(2 \delta-\Delta-1)$.

СлеДСТВИЕ. Пусть $H-$ псевдограф с матрицей инцидентности $A=A(H) \in$ $\mathfrak{A}^{m, n}(\leqslant 2,[\delta, \Delta])$ и числом независимости $\mu$. Тогда в $i$-канонической форме $A$ число строк $m_{3}$ в подматриче $(A)_{3}$ не меньше, чем $\mu(2 \delta-\Delta-2) / 2$, а если $\Delta$ нечетно, то существует такой $i$-базис (т.е. такое паросочетание в псевдографе), что в $і$-канонической форме относительно этого базиса $m_{3} \geqslant(2 \delta-\Delta-1) / 2$.

Теперь мы можем получить нижнюю гранищу числа независимости псевдографа. 
Теорема 2. Пусть $H$ - псевдограф с п вершинами, $m$ ребрами, максимальной степенью вершины $\Delta$ и минимальной степенью вериины $\delta, \mu-е г о$ (реберное) число независимости. Предположим, что $\delta \geqslant(\Delta+2) / 2$. Тогда если $\Delta$ нечетно, mo

$$
\mu(H) \geqslant \frac{2}{2 \delta+\Delta-1}(\delta n-m) ;
$$

при четном $\Delta$ справедливо неравенство

$$
\mu(H) \geqslant \frac{2}{2 \delta+\Delta}(\delta n-m)
$$

ДокАЗАТЕЛЬСтво. Пусть $A(H)$-матрища инцидентности псевдографа $H$. Тогда $A(H) \in \mathfrak{A}^{m, n}(\leqslant 2,[\delta, \Delta])$ и ее $i$-ранг равен $\mu$. Пусть $A$ - $i$-каноническая форма $A(H)$ относительно $i$-базиса $\mathscr{L}$. При нечетном $\Delta$ предположим, что $\mathscr{L}$ взят таким образом, чтобы для числа единищ в подматрище $(A)_{3}$ матрицы $A$ вьполнялось неравенство (4). По построению $i$-канонической формы число строк в $(A)_{3}$ не может быть больше, чем $m-\mu-\delta(n-2 \mu)$. Поэтому

$$
\sigma_{1}\left((A)_{3}\right) \leqslant 2[\mu(2 \delta-1)-(\delta n-m)]
$$

Пусть $\Delta$ нечетно. Предположим от противного, что

$$
\mu<\frac{2}{2 \delta+\Delta-1}(\delta n-m)
$$

следовательно,

$$
\delta n-m>\frac{2 \delta-\Delta-1}{2} \mu .
$$

Оценивая $\delta n-m$ согласно (9), из (8) получаем, что

$$
\sigma_{1}\left((A)_{3}\right)<(2 \delta-\Delta-1) \mu
$$

что противоречит неравенству (4) теоремы 1.

Совершенно аналогично, при $\Delta$ четном предположение о том, что

$$
\mu<\frac{2(\delta n-m)}{2 \delta+\Delta}
$$

приводит к противоречию с неравенством (3). Теорема доказана.

Заметим, что если $\delta>(\Delta+2) / 2$, то неравенство (7) при $\delta>2$ и неравенство (6) дают лучшие нижние границы числа независимости псевдографа, чем общая оценка (1) в теореме А. Эти границы могут быть использованы при изучении числа реберного покрытия гиперграфов при ограничениях на степени его вершин. 
3. Гипотеза Райзера. В этом пункте мы применим оценку (1) числа независимости гиперграфа к изучению гипотезы Райзера для $r$-дольных $r$-графов (неравенство (2)). При $r=2$ (т.е. для графов) это неравенство справедливо в силу классического комбинаторного результата - теоремы Кёнига-Эгервари, и гипотезу можно рассматривать как обобщение этого неравенства на $r$-дольные $r$-графы при произвольном $r \geqslant 2$. Неравенство (2) доказано лишь в следуюших частных случаях:

1) $r=3$ и $\mu(H) \leqslant 4$;

2) $r=4$ и $\mu(H) \leqslant 2$;

3) $r=5$ и $\mu(H)=1$

(см. [4]). Наиболее часто рассматривался случай $r=3$. Были получены оценки вида $\tau(H) \leqslant \alpha \mu(H), 2<\alpha<3$, усиливающие очевидную оценку $\tau(H) \leqslant 3 \mu(H)$, наилучшая среди них:

$$
\tau(H) \leqslant 2.5 \mu(H)
$$

для любого 3 -дольного 3 -графа $H[5]$.

Ясно, что во всяком $r$-дольном $r$-графе с $n$ вершинами имеются трансверсали $T$, состоящие из $n / r$ вершин. Поэтому имеем неравенство

$$
\tau(H) \leqslant \frac{n}{r}
$$

для любого $r$-дольного $r$-графа с $n$ вершинами. Мы будем использовать лишь эту простую оценку (11) для $\tau(H)$.

Фиксируем натуральные числа $r$ и $\delta, m$ и $n$. Рассмотрим гиперграфы $H$ с $m$ ребрами, $n$ вершинами, для которых $r(H) \leqslant r, \delta(H) \geqslant \delta$. Оценивая число единиц в матрице инцидентности такого гиперграфа, нетрудно убедиться в том, что

$$
\delta n \leqslant r m .
$$

Если при этом каждое ребро $H$ состоит из $r$ вершин и каждая вершина принадлежит в точности $\delta$ ребрам (т.е. $H-\delta$-регулярный $r$-граф), то неравенство (12) обращается в равенство

$$
\delta n=r m .
$$

Матрица инцидентности $\delta$-регулярного $r$-графа есть $(0,1)$-матрица класса $\mathfrak{A}^{m, n}(r, \delta)$, состоящего из всех $(0,1)$-матриц размера $m \times n$ со строчными суммами $r$ и столбцовыми суммами $\delta$; нетрудно показать, что этот класс непуст при любых $m, n, r, \delta$, для которых выполняется равенство (13). Класс $\delta$-регулярных $r$-графов обозначим через $\mathscr{U}(r, \delta)$. Нетрудно убедиться, рассматривая соответствующие матрицы инцидентности, что при $r \mid n$ в $\mathscr{U}(r, \delta)$ всегда найдутся $r$-дольные гиперграфы. Класс $r$-дольных $\delta$-регулярных $r$-графов обозначим через $\mathscr{P} \mathscr{U}(r, \delta)$.

TЕОРема 3. Пусть $r \geqslant 3, \delta \geqslant 2$ - натуральные числа, $H$ - гиперграф из $\mathscr{P} \mathscr{U}(r, \delta)$, $\tau(H), \mu(H)$ - соответственно трансверсальное число и число независимости гиперграфа $Н$. Для $Н$ справедлива гипотеза Райзера

$$
\tau(H) \leqslant(r-1) \mu(H),
$$

если только $\delta \leqslant r-1$. 
ДокАЗАТЕЛьство. Пусть гиперграф $H$ имеет $n$ вершин и $m$ ребер. Тогда вьполнено (13). Применяя к $H$ теорему А, оценим снизу число независимости $\mu(H)=\mu$ :

$$
\mu \geqslant \frac{\delta n-(r-1) m}{\delta r-r+1}=\frac{\delta n}{r(\delta r-r+1)} .
$$

Имеем, очевидно, $\delta r-r+1>0$. Поэтому из (11) и (14) следует, что

$$
\tau(H)=\tau \leqslant \frac{n}{r} \leqslant \frac{\delta r-r+1}{\delta} \mu .
$$

По условию $\delta \leqslant r-1$, поэтому $\delta r-\delta \geqslant \delta r-r+1$ и $(\delta r-r+1) / \delta \leqslant r-1$. Таким образом, находим, что $\tau \leqslant(r-1) \mu$. Теорема доказана.

ТЕОРемА 4. Пусть $H-r$-дольный $r$-граф с $n$ вершинами и т ребрами и $m=n$. Предположим также, что максимальная степень $\Delta$ вершины гиперграфа $H$ не превосходит $r$. Тогда для любого $r \geqslant 2$ справедлива оченка

$$
\tau(H) \leqslant(r-1) \mu(H)+\frac{\mu(H)}{r},
$$

әде $\tau(H)=\tau, \mu(H)=\mu-$ соответственно трансверсальное число и число независимости гиперграфа $H$.

ДокАЗАТЕЛЬСТво. Пусть $\delta$ - минимальная степень вершины в $H$. Тогда выполнено (12). Рассмотрим матрицу инцидентности $A(H)$ гиперграфа $H$. Число единиц в ней равно $r m$, т.е. $r n$ по условию. Отсюда следует, что $\delta \leqslant r$. Однако, если степень хотя бы одной вершины будет меньше, чем $r$, то ввиду условия $\Delta \leqslant r$ число единиц в матрице не может достигнуть $r n$. Это показывает, что $\delta=\Delta=r$ и $H r$-регулярен. Таким образом, оценка (1) в применении к $H$ дает

$$
\mu \geqslant \frac{n}{r^{2}-r+1}
$$

Отсюда получаем, что

$$
\frac{n}{r} \leqslant \frac{r^{2}-r+1}{r} \mu
$$

и ввиду (11)

$$
\tau \leqslant(r-1) \mu+\frac{\mu}{r}
$$

что и требовалось доказать.

СлЕДСТВИЕ. Для $r$-дольного $r$-регулярного $r$-графа справедливо

$$
\tau(H)=(r-1) \mu(H),
$$

если $n<r^{2}$, әде $n$ - число вершин в $H$.

Действительно, в этом случае $\mu<r, \mu / r<1$.

Заметим, что при $r=3$ из (15) получается оценка $\tau(H) \leqslant(2+1 / 3) \mu(H)$, улучшающая для гиперграфов из $\mathscr{P} \mathscr{U}(3,3)$ общую оценку $(10)$ для произвольного 3 -регулярного 3-графа.

В заключение сделаем еще два замечания, касающиеся общего случая. 
ЗАмечаниЕ 1. Нетрудно заметить, что для нерегулярных $r$-дольных $r$-графов $H$ с $n$ вершинами и $m$ ребрами, у которых отношение $m / n$ достаточно близко к $\delta(H) / r(H)$, с помощью (1) также получаются оценки вида $\tau \leqslant \alpha \mu \mathrm{c} r-1<\alpha<r$. Так, например, для 3 -дольных 3 -графов при $\delta=2$ и $2 / 3<m / n<11 / 15$ находим, что $\tau \leqslant \alpha \mu$ с $\alpha<2.5$.

ЗАмЕчАниЕ 2. При рассмотрении $r$-дольных $r$-графов $H=(V, E)$ с $|V|=n$ мы использовали лишь наиболее простые, заведомо сушествуюшие в каждом таком гиперграфе трансверсали $T$ с $|T|=n / r$. Существование трансверсали $T$ с $|T|=\beta n, \beta<1 / r$, дает возможность с помощью (1) получать более точные оценки трансверсального числа через число независимости.

Математический институт

Поступило

им. В. А. Стеклова РАН

19.09 .96

\section{СПИСОК ЦИТИРОВАННОЙ ЛИТЕРАТУРЫ}

[1] Тараканов В. Е. О максимальной глубине одного класса $(0,1)$-матриц // Матем. сб. 1968. T. $75(117)$. C. $4-14$.

[2] Тараканов В. Е. Максимальная глубина произвольных классов $(0,1)$-матриц и некоторые ее применения // Матем. сб. 1973. Т. 92(134). С. 472-490.

[3] Bollobás B. Extremal graph theory. London: Acad. Press, 1978.

[4] Tuza Zs. Ryser's conjecture for transversals of $r$-partite hypergraphs // Ars Combin. 1983. V. 16 (B). P. 201-209.

[5] Haxell P. E. A note on a conjecture of Ryser // Period. Math. Hungar. 1995. V. 30. №1. P. 73-79.

[6] Райзер Г. Дж. Комбинаторная математика. М.: Мир, 1966. 